\title{
Study on the formability by TPIF technology for aluminium sheet at room temperature
}

\author{
Ma Van Viet ${ }^{1}$, Nguyen Truong Thinh ${ }^{1}$, Le Van $\mathrm{Sy}^{2 *}$, and Svetlin Antonov ${ }^{3}$ \\ ${ }^{1} \mathrm{HCMC}$ University of Technology and Education, Department of Mechanical Engineering, Vietnam \\ ${ }^{2}$ PetroVietnam University, Ba Ria city, Vietnam \\ ${ }^{3}$ Technical university of Sofia, Faculty of Telecommunications, Bulgaria
}

\begin{abstract}
Two Point Incremental Forming technology (TPIF) is one forming method of incremental sheet forming technology (ISF) which is an innovation sheet forming process with potential advantages such as simplicity, less-time consumption, and high flexibility. This technology using a hemispherical-end tool under CNC movement deforms a metal sheet which is fixed on simple frame. The sheet metal clamped between movable plate and clamp plate, under the metal sheet has a support die which is fixed on bottom plate. The lower plate is firmly positioned on the $\mathrm{CNC}$ machine table in while upper plate (included sheet material, movable plate and clamp plate) is able to move easily up and down along guide bars. The sheet material is plastically deformed layer by layer until finalshape product by $\mathrm{CNC}$ tool path. This technology is very suit for the rapid prototyping process and the low batch production. In this research, formability of the TPIF process due to operating parameters was investigated with aluminum sheet at room temperature. Four operating parameters such as depth step, feed rate, tool diameter, and spindle speed, was considered their effects on the formability of TPIF process through DOE strategy. The forming results showed that TPIF process for metal sheet material at room temperature has potential applicability in the metal sheet-product manufacturing.
\end{abstract}

\section{INTRODUCTION}

Incremental sheet forming, an invention of Leszak in 1967, sheet material (metal and polymer sheet), has been researched the focus of many studies. This process uses a forming tool fixed on a 3-axis CNC milling machine, is controlled by a toolpath. A material is fixed on a simple frame by bolts, is deformed layer by layer by a head of forming tool. The toolpath is exported from the complete geometry of the product through a traditional CAM software. The ISF method is two kinds of Single Point Incremental Forming (SPIF) and Two Point Incremental Forming (TPIF). TPIF and SPIF jig structure differents are a sheet metal of TPIF can move up and down along guide bars, under sheet metal of TPIF has got a support which is fixed died on base plate (fig.1). TPIF is an innovation sheet forming process with potential advantages such as simplicity, less-time consumption, and high

\footnotetext{
*Corresponding author: sylv@pvu.edu.vn
} 
flexibility. This technology using a hemispherical-end tool under CNC movement deforms a metal sheet which is fixed on simple frame (movable plate). The sheet metal clamped between movable plate and clamp plate, under the metal sheet has a support die which is fixed on base plate which is firmly positioned on the CNC machine table in while upper plate (included sheet material, movable plate and clamp plate) is able to move easily up and down along guide bars. The sheet material is plastically deformed layer by layer until finalshape product by CNC toolpath. This technology is very suit for the rapid prototyping process and the low batch production. A review of TPIF researches such as some topics.

H. Meier et al [8] used two industrial robots for TPIF, compared to other incremental sheet metal forming machines, this system offers a high geometrical form flexibility without the need of any workpiece dependent tools. This way, the surface quality improved highly.

J. Jeswiet et al [13] compare forces in SPIF and TPIF, The forces measured in forming cones and truncated pyramids from 3003-0 Aluminum sheet, $1.21 \mathrm{~mm}$ thick, the forces for SPIF and TPIF are the same magnitude. A. Attanasio et al [14] do experiments on a car door handle cavity for evaluating geometrical and dimensional errors, and surface finishing, between TPIF and SPIF with the same working parameters. TPIF assures the achievement of a better dimensional accuracy and surface finishing. Isabel Bagudanch et al [7] investegate a truncated pyramid frustum and a circular generatrix with parameters (step down, tool diameter, feed rate and spindle speed), PVC and PC sheet material between TPIF and SPIF. TPIF is geometrical accuracy and reduce the effect of the springback. So, TPIF technology is better geometrical accuracy and surface quality than SPIF.

Seyed Ali Asghar Asghari et al [9]. were optimate by grey relational analysis with response factors (Min thickness, Springback, Surface roughness) optimize parameters were $15 \mathrm{~mm}$ tool nose diameter, $63^{\circ}$ wall angle, $800 \mathrm{r} / \mathrm{min}$ spindle speed and $0.2 \mathrm{~mm}$ deep step with cone shape, analyze formability of aluminum 1050 in TPIF. M. Safari [15] investigated a complicated shape with positive and negative truncated cones, aluminum alloy 3105, 1mm thickness by TPIF with step depth, rotational speed. An optimum parameter combination (Negative/Positive, step depth $0.2 \mathrm{~mm}$ and rotational speed 1000 rpm) is obtained to get both maximum achievable outer and inner heights using signal to noise ratio analysis. Hani Mostafanezhad et al [16] studied experimental study based on response surface methodology (RSM) was carried out to analyze effect of wall angle, tool nose diameter, initial sheet thickness and step down on thinning ratio and forming force during TPIF of AA1050 truncated cone. A series of experiments was carried out based on Box-Bhenken experimental design and mathematical models of responses are developed by means of RSM and nalysis of variances. Response surface methodology optimal parameter setting regarding minimum thinning ratio and forming force.

Numerical simulation, Chenhao Wang et al [17] study The enhanced Lemaitre damage model accounting for the micro-crack closure effect is adopted to predict the fracture in TPIF by using Abaqus/Explicit subroutine VUMAT. The material constants in the damage model are calibrated throughout tensile tests by minimizing the force error using Newton approach. The TPIF with a hemispherical shape using the enhanced Lemaitre CDM damage model in FEM shows a good agreement of the thickness distribution, fracture depth and the forming force trend compared with the corresponding experimental results. It is concluded that the enhanced CDM-based Lemaitre model can be used for ductile fracture of AA 7075 aluminium alloy in TPIF with a hemispherical shape. R. Perez-Santiago et al [18] investigate force is a more rigorous process parameter for validation of FEM models qualitative trends like thickness distribution and higher forces obtained at higher $\Delta \theta$ are correctly reproduced. Adil Shbeeb Jaber et al [19] study forming mechanism and multi stages incremental forming.step size and forming tool radius, on the thickness distribution and strain analyses for three stages in multi, with vertical angle.2-D model of cone shaped 
part with right forming angle with a wall angle of $60^{\circ}$, thickness $(1 \mathrm{~mm})$ of the aluminum alloy (AA1070). ANSYS 11 software is used to carry out the numerical simulation of the multistage. The results show that, when considering multi-stage incremental sheet forming, the task is even more difficult because the strain and thickness distribution resulting from the first stage will influence the subsequent results. Decreasing in the forming tool radius will increase in the thinning of the wall product due to excessive stretch will occurs, while the incremental step size is not significant effect on the numerical results (thickness, strain) distribution of the product. Finally, the goal to attain a vertical wall angle and equally maintain wall thickness and strain over the wall part is pursued. Mechanical tests, computer programming, geometry and design were required. The simulation results including the thickness and strain distributions over the product walls throughout three stages were concluded. Haibo Lu et al [20] study Part accuracy improvement in two point incremental forming with a partial die using a model predictive control algorithm, a non-axisymmetric shape, which contains both flat and curved walls, The wall angle was $40^{\circ}, 35 \mathrm{~mm}$ depth, aluminium (AA 7075-O), $1.6 \mathrm{~mm}$ thickness, $20 \mathrm{~mm}$ tool diameter, feed rate $4000 \mathrm{~mm} / \mathrm{min}$. The control algorithm toolpath correction in the horizontal and vertical directions through optimising two toolpath parameters $\left(\Delta u_{r}\right.$ and $\left.\Delta u_{z}\right)$ in two separate control modules. Compared with the typical, TPIF process that has no toolpath correction, fairly good improvement in geometric accuracy was achieved with the use of the toolpath correction strategy in TPIF with a partial die while the geometric accuracy in the partial fillet areas requires further improvement. This work provides a helpful approach to achieve in-process toolpath control/correction in TPIF.

Xiaoqiang LI et al [21] study experimental and numerical investigation on surface quality for two-point incremental sheet. Forming with interpolator, the influences of process variables (i.e. tool diameter, step size and thickness of interpolators) on the forming process (e.g. surface roughness, forming force and geometric error) are investigated through a systematic experimental approach of central composite design (CCD) in twopoint incremental sheet forming (TPIF). The increase in thickness of interpolators decreases the surface roughness in direction vertical to the tool path while increases the surface roughness in direction horizontal to the tool path. The combined influence between thickness of interpolators and process parameters (tool diameter and step size) is limited. The placement of interpolator has little influence on the effective forming force of blank. The geometric error enlarges with the increase of step size and thickness of interpolator while decreases firstly and then increase with an increase in tool diameter. The influencing mechanism of the interpolator method on surface quality can be attributed to the decrease of the contact pressure due to the increase of contact area with the unchanged contact force.

Although the research teams have studied improve surface quality, compare between SPIF and TPIF, geometrical accuracy, response surface methodology optimal parameter setting regarding minimum thinning ratio and forming force, numerical simulation, etc. on TPIF, the formability of sheet material has not been investigated clearly. Therefore, this paper will focus on the formability of sheet material such as aluminum sheet A 1050 H14. The formability of sheet material is investigated by experiment with results towards investigation of maximum wall angle of aluminum sheet A $1050 \mathrm{H} 14$, thickness of $1.5 \mathrm{~mm}$. In this research, a step frustum cone shape with $1^{\circ}$ for every step (investigated angle from $65^{\circ}-85^{\circ}$ ) is used to investigate formability of the TPIF process due to operating parameters was investigated with aluminum sheet at room temperature. This investigated shape is a new model in study on the formability by TPIF technology. It has never been used the last researches. Four operating parameters such as spindle speed, depth step, feed rate, tool diameter were considered their effects on the formability of TPIF process through DOE strategy. The forming results showed that TPIF process for metal sheet material at room temperature has potential applicability in the metal sheet-product manufacturing. 


\section{EXPERIMENTAL EQUIPMENTS}

In this study, the jig/fixture for TPIF process is designed to form aluminum sheet at room temperature. The jig/fixture system consists of four guide bars which fix die on a base plate. A support die is fixed on a base plate by four bolts. Four Linear Bushings are fixed die on a movable plate; the group (linear bushing, movable plate) fix on guide bars with linear bushing and can move up and down along guide bars. Between a movable plate and a clamp plate, there is a sheet material is clamped by eight bolts (figure 1). Dimensions of the metal sheet are $400 \times 400 \mathrm{~mm}^{2}$ and 1.5 thickness. The jig/fixture is clamped on the CNC milling machine table (Figure 2). The forming tool has a hemispherical-end shape with diameter equal to $6 \mathrm{~mm}, 12 \mathrm{~mm}, 18 \mathrm{~mm}$ which is always pressed into the metal sheet surface to create a locally plastic deformation. In this system, the forming tool is designed with enough length to form complete product. It is made from steel round bar and steel ball was welded on the top of the bar to ensure good hardness and wear resistance (Figure 3). The forming tool is checked inversion (figure 4). Mixed lubrication is solid graphite powder and Lithium grease with scale 1:1 and lubrication oil (multi 20W-50) to create linked mixed lubrication, it is used in the experiments to reduce the contact friction between the forming tool and the metal sheet surface.

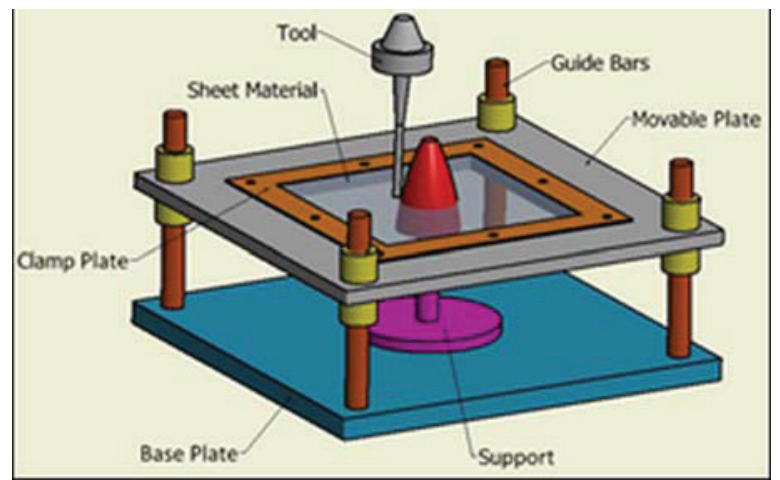

Fig. 1. CAD model of Jig and fixture system for fixture system for TPISF process.

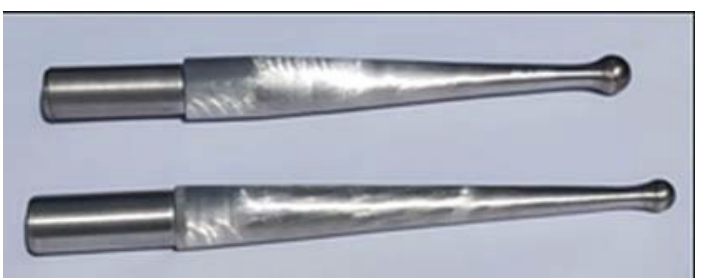

Fig.3 Forming tool in the experimental work.

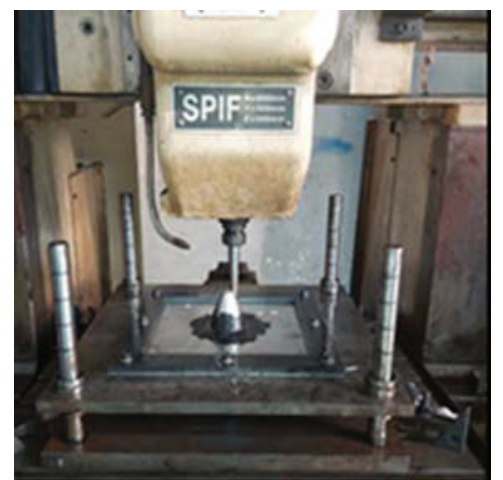

Fig.2 Practical model of Jig and TPIF process.

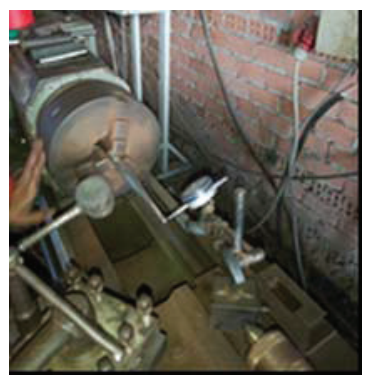

Fig.4 The forming tool is checked inversion

\section{EXPERIMENTAL DESIGN}


The experiments are performed with a step frustum cone shaped product (Figure 5), which has a step frustum cone to realize the influence of the processing parameters on the formability of aluminum sheet A $1050 \mathrm{H} 14$ at room temperature. The product profile with a step frustum cone shaped enables the investigation of all the from $65^{\circ}$ to $85^{\circ}$ (Fig. 6). The slope of the profile increases with its height, the analyzed region is limited to an angle less than $85^{\circ}$. An experimental strategy is planned based on the DOE approach to determine the influence of the processing parameters on the formability of aluminum sheet at room temperature.

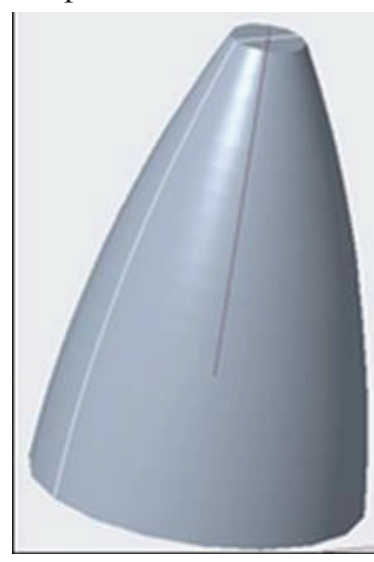

Fig.5 CAD model

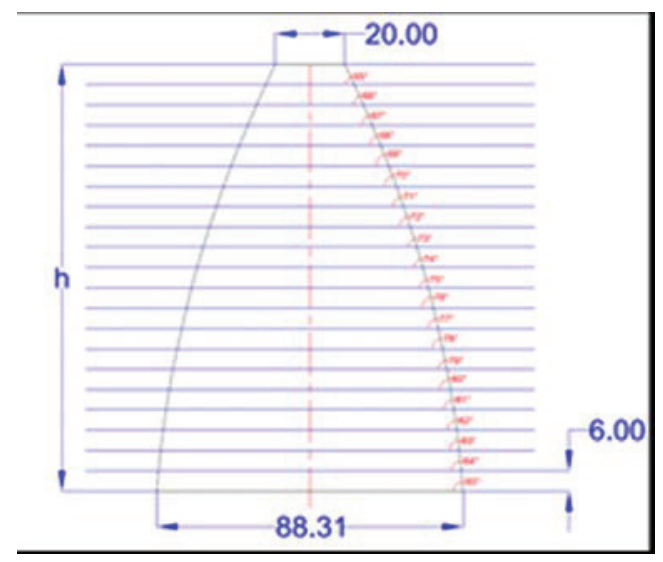

Fig.6 Profile of a shape cone model.

The forming parameters such as depth step $(\Delta \mathrm{z})$, feed rate $(\mathrm{V}$-xy), tool diameter (D), and spindle speed (n) are chosen to investigate formability of metal sheets based on previous studies [7]. The Box-Behnken, 5 center points design was applied. Minitab 19 software was used based on the selected factors and values in Table 1. A design matrix with 29 experimental runs was generated. Response parameter is maximum wall angles.

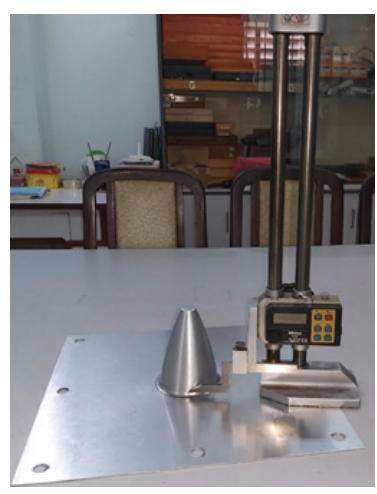

Fig.7 Measurement of mechanical failure height.
Table 1. Processing parameters for experimental design.

\begin{tabular}{|c|l|c|c|c|c|c|}
\hline & & & & \multicolumn{3}{|c|}{ Range of values } \\
\cline { 4 - 7 } No & $\begin{array}{l}\text { Experiment } \\
\text { al } \\
\text { parameters }\end{array}$ & $\begin{array}{c}\text { Sym } \\
\text { boy }\end{array}$ & Unit & $\begin{array}{c}\text { Low } \\
\text { level }\end{array}$ & $\begin{array}{c}\text { Midiu } \\
\mathrm{m} \\
\text { level }\end{array}$ & $\begin{array}{c}\text { High } \\
\text { level }\end{array}$ \\
\hline 1 & Depth step & $\Delta \mathrm{z}$ & $\mathrm{mm}$ & 0.1 & 0.8 & 1.5 \\
\hline 2 & Feed rate & $\mathrm{Vxy}$ & $\begin{array}{l}\mathrm{mm} / \\
\mathrm{minu}\end{array}$ & 300 & 900 & 1500 \\
\hline 3 & $\begin{array}{l}\text { Tool } \\
\text { diameter }\end{array}$ & $\mathrm{D}$ & $\mathrm{mm}$ & 6 & 12 & 18 \\
\hline 4 & $\begin{array}{l}\text { Spindle } \\
\text { speed }\end{array}$ & $\mathrm{n}$ & $\mathrm{rpm}$ & 300 & 1050 & 1800 \\
\hline
\end{tabular}




\section{RESULTS AND DISCUSSION}

The deformation ability of sheet material is measured by wall angle ( ). The higher wall angle is, the greater formability of sheet metal is. The wall angle is measured continuously through the height $(\mathrm{h})$ of the mechanical failures from head of a step frustum cone to mechanical failures on the product (Figure 7). This values are converted into maximum wall angles and insert into the design matrix. According to the experiment results, the roughness of the surface in direct contact with the forming tool is smaller than the other surface. Due to many different factors such as lubrication conditions, contacting condition, machine parameters, etc. Therefore, these parameters are controlled to increase surface quality.

Using the Minitab 19 software and the experimental data, we have the ANOVA for response as Table 2 . The p-value is less than 0.05 , model is suitable statistically significant. The analysis of variance (ANOVA) shows that the percent influence of parameters and the interaction parameters to the effects on formability of sheet metal. Percent contribution of total variance such as depth step (A) $22.46 \%$, feed rate (B) $18.59 \%$, tool diameter (C) $26.77 \%$, spindle speed (D) $0.19 \%$, AA $22.63 \%$, AC $5.02 \%$, BC $0.56 \%$. The coefficient of determination for regression analysis (R-squared (R2)) is 0.9625 is the goodness-of-fit of the model to the experimental data. It is very close to 1 , and obtain $96.25 \%$ of the total variance.

Table 2. The Analysis of Variance (ANOVA)

\begin{tabular}{|c|r|r|r|r|r|c|}
\hline Source & DF & Adj SS & Adj MS & $\begin{array}{r}\text { F- } \\
\text { Value }\end{array}$ & $\begin{array}{r}\text { P- } \\
\text { Value }\end{array}$ & $\%$ \\
\hline Model & 8 & 43.1462 & 5.3933 & 64.15 & 0.000 & 96.25 \\
\hline $\mathrm{z}$ & 1 & 10.0833 & 10.0833 & 119.94 & 0.000 & 22.46 \\
\hline $\mathrm{V}$-xy & 1 & 8.3333 & 8.3333 & 99.13 & 0.000 & 18.59 \\
\hline $\mathrm{n}$ & 1 & 0.0833 & 0.0833 & 0.99 & 0.331 & 0.19 \\
\hline $\mathrm{D}$ & 1 & 12.0000 & 12.0000 & 142.74 & 0.000 & 26.77 \\
\hline $\mathrm{z}^{*} \mathrm{z}$ & 1 & 10.1462 & 10.1462 & 120.69 & 0.000 & 22.63 \\
\hline $\mathrm{z}^{*} \mathrm{D}$ & 1 & 2.2500 & 2.2500 & 26.76 & 0.000 & 5.02 \\
\hline Error & 20 & 1.6814 & 0.0841 & - & - & 3.75 \\
\hline Total & 28 & 44.8276 & - & - & - & - \\
\hline
\end{tabular}

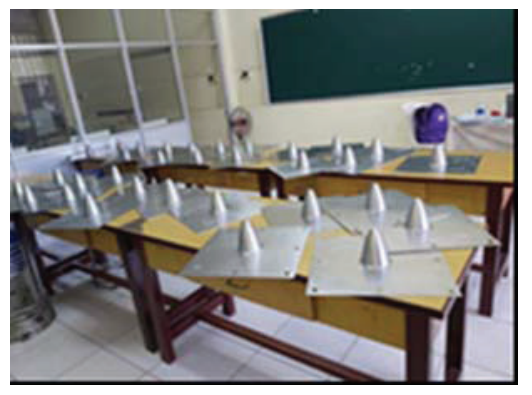

Fig. 8 The Products by TPIF Technology.

\begin{tabular}{rrrr} 
S & R-sq & R-sq(adj) & R-sq(pred) \\
\hline 0.289946 & $96.25 \%$ & $94.75 \%$ & $88.96 \%$
\end{tabular}

Regression models for most significant parameters on responses presenting a relationship among processing parameters and their interactions are shown below

$\alpha=79.170+7.374 \mathrm{z}+0.000556 \mathrm{~V}-\mathrm{xy}+0.000111 \mathrm{n}-0.0863 \mathrm{D}-2.451 \mathrm{z}^{*} \mathrm{z}+$ $0.000000 \mathrm{z} * \mathrm{~V}-\mathrm{xy}-0.1786 \mathrm{z} * \mathrm{D}+0.000069 \mathrm{~V}-\mathrm{xy} * \mathrm{D}$

According to correlation equation, it shows that tool diameter (D) is inversely proportional to the wall angle. The wall angle is proportional to depth step $(\Delta \mathrm{z})$ and feed rate $(\mathrm{V}-\mathrm{xy})$.

The result of numerical optimization with maximum wall angle is 84.3625 degree. 


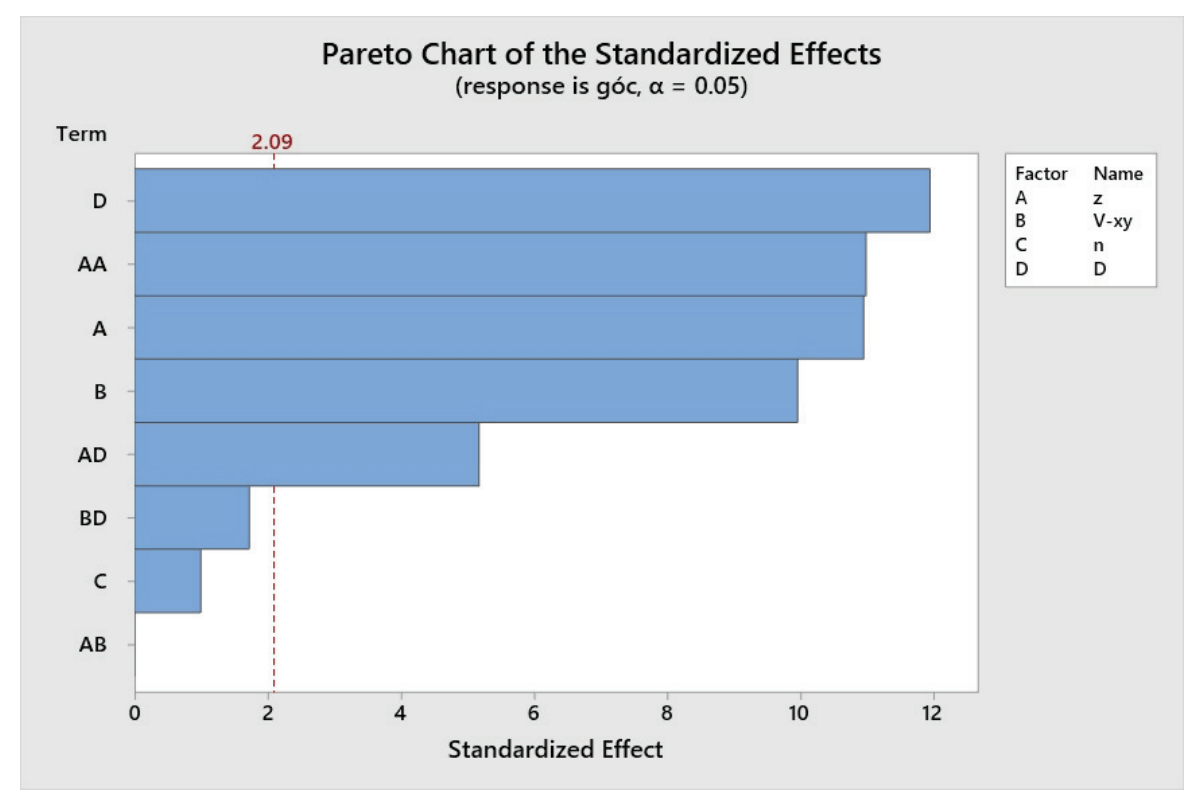

Fig. 9 Pareto Chart for wall angle.

According to Pareto chart for wall angle (Figure 9), it shows that important factors in descending order as tool diameter (D), depth step interactive to depth step $\left(z^{*} \mathrm{z}\right)$, depth step $(\mathrm{z})$, and feed rate (Vxy).

\section{CONCLUSIONS}

The Jig and fixture system for TPISF process is designed to fix aluminum sheet A 1050 $\mathrm{H} 14,1.5 \mathrm{~mm}$ thickness for investigating the influences of processing parameters on formability, surface quality at room temperature.

The maximum wall angle $\left(84^{\circ}\right)$ achieved in TPIF with aluminum sheet A $1050 \mathrm{H} 14,1.5$ mm thickness at room temperature.

According to the response wall angle analysis, the predicted result of the model is reasonable alignment with the observations taken from the experiments. Thus, the established model can be utilized to estimate the wall angle in TPIF process with $96.25 \%$ confidence within the range of investigated machining conditions.

Optimized maximum wall angle is 84.3625 degree.

The percentage error between the experimental and predicted values of the minimum wall angle is $3.75 \%$, and is found to be insignificant.

Reduce friction between the tool and the sheet metal by good lubrication to have the surface quality. The roughness of the surface in direct contact with the forming tool is smaller than the other surface.

This research is supported by Key Laboratory of Digital Control and System Engineering (DCSELAB), HCMUT, VNU-HCM under grant number TX2020-20b-01. 


\section{References}

1. Le Van Sy, "modeling of single point incremental forming process for metal and polymeric sheet," University of Padua, Department of innovation in Mechanics and Management, 2009.

2. G. Yanle Li \& Xiaoxiao Chen \& Zhaobing Liu \& Jie Sun \& Fangyi Li \& Jianfeng Li \& Guoqun Zhao, "A review on the recent development of incremental sheet-forming process," Springer-Verlag London, March 2017.

3. M. B. Silva and P.A.F. Martins, "Two Point Incremental Forming with partial die: theory and experimentation," JMEPEG, no. 22, pp. 1018-1027, 2013.

4. L. T. De Luca, Propulsion physics (EDP Sciences, Les Ulis, 2009) Hani Mostafanezhad, Hossein Ghorbani Menghari, Samad Esmaeili, EhsanMarzban Shirkharkolaee, "Optimization of Two-point incremental forming process of AA1050 through response surface methodology," Measurement, 2018.

5. Le Van Sy, Nguyen Thanh Nam, "Hot Incremental Forming Of Magnesium And Aluminum Alloy Sheets By Using Direct Heating System.: Proceeding of the Institution of Mechanical Engineers," Engineering Manufacture, no. Part B, 2013.

6. V. Sy Le, Ghiotti A and Lucchetta G. Preliminary studies on single point incremental forming for thermoplastic materials. Int J Mater Form 2008; 1(1): 1179-1182.

7. I. Bagudanch, Marc Sabater \& Maria Luisa Garcia-Romeu, "Single Point versus Two Point Incremental Forming of thermoplastic materials," Advances in Materials and Processing Technologies, no. 2374-068X (Print), pp. 2374-0698.

8. H. Meiera, V. Smukalab, O. Dewaldc and J. Zhangd, "Two Point Incremental Forming with two moving forming tools, " Key Engineering Materials, Trans Tech Publications, Switzerland., vol. 344, no. 1662-9795, pp. 599-605, 2007.

9. S. Ali Asghar Asghari, Asghar Shamsi Sarband and Mostafa Habibnia, "Optimization of multiple quality characteristics in two-point incremental forming of aluminum 1050 by grey relational analysis," Institution of Mechanical Engineering Science, vol. Proc IMechE Part C, 2017.

10. A. Dabwan, Adham E. Ragab, Mohamed A. Saleh, Saqib Anwar, Atef M. Ghaleb, and Ateekh Ur Rehman, Study of the Effect of Process Parameters on Surface Profile Accuracy in Single-Point Incremental Sheet Forming of AA1050-H14 Aluminum Alloy, Hindawi Advances in Materials Science and Engineering Volume 2020, Article ID 7265941, 14 pages.

11. N. Thanh Nam, Phan Dinh Tuan, Vo Van Cuong, Le Khanh Dien, research on the forming angle of A1050-H14 aluminum material processed by using single point incremental forming technology (SPIF), Science \& Technology Development, Vol 12, No.16 - 2009

12. A. Al-Shayea, Abdulmajeed DABWAN, Adham E RAGAB, Mustafa M. NASR, Husam KAID, Studying the Effect of Process Parameters on Part Depth in Single Point Incremental Forming of AA1050-H14 Aluminum Alloy Sheets, ISSN 1330-3651 (Print), ISSN 1848-6339.

13. J. Jeswiet, J. R. Duflou and A. Szekeres, "Forces in Single Point and Two Point Incremental Forming," Advanced Materials Research, vol. 6-8, pp. 449-456, 2005

14. A. Attanasio, E. Ceretti, L. Mazzoni, C. Giardini, "Use of TPIF or SPIF for Prototype Productions, "Conference on Material Forming, American Institute of Physics, pp. 978-0-7354-0414-4, 2007. 
15. M. Safari, Two Point Incremental Forming of a Complicated Shape with Negative and Positive Dies, IJMF, Iranian Journal of Materials Forming, Vol. 4, No. 2, pp 51-61 Printed in The Islamic Republic of Iran, 2017.

16. H. Mostafanezhad1, Hossein Ghorbani Menghari, Samad Esmaeili, Ehsan Marzban Shirkharkolaee, Optimization of Two-point incremental forming process of AA1050 through response surface methodology, Measurement (2018), doi: https://doi.org/10.1016/j.measurement.2018.04.042.

17. C. Wang, William J.T. Daniel, Haibo Lu, Sheng Liu, Paul A. Meehan, "FEM Investigation of Ductile Fracture Prediction in Two-Point Incremental Sheet Metal Forming process, ScienceDirect, Procedia Engineering.," 2017, no. 207 , pp. 836-841.

18. R. Perez-Santiago, A. Fiorentino, R. Marzi, and C. A. Rodriguez, "Advances in simulation of Two Point Incremental Forming," AIP Conference Proceedings, vol.183, p.1353, 2011.

19. A. Shbeeb Jaber, "Finite Element Simulation Of The Two Point Multistage Incremental Sheet Metal Forming Process,"Engineering and Sustainable Development, vol. 21, no. 5, September 2017

20. H. Lu, Michael Kearney, Chenhao Wang, Sheng Liu, Paul A. Meehan, "Part accuracy improvement in two point incremental forming with a partial die using a model predictive control algorithm," Accepted Manuscript.

21. X. Li, Kai Han, Xu Song, Haibo Wang, Dongsheng Li, Yanle Li, Qing Li "Experimental and numerical investigation on surface quality for two-point incremental sheet forming with interpolator," Chinese Journal of Aeronautics, Pages 13, February 2020. 